\title{
Aged Human Skin is More Susceptible than Young Skin to Accumulate Advanced Glycoxidation Products Induced by Sun Exposure
}

Pageon $\mathrm{H}^{*}$, Poumès-Ballihaut $\mathrm{C}$, Zucchi $\mathrm{H}$, Bastien $\mathrm{P}$, Tancrede $\mathrm{E}$ and Asselineau D

L'Oréal Research \& Innovation, Aulnay-sous-bois, France

\begin{abstract}
Background: Skin aging is the result of intrinsic and extrinsic phenomena. Among the factors involved in skin aging, the glycation reaction and the formation of advanced glycation end-products (AGEs) is an important element. AGEs such as carboxy-methyl-lysine (CML) and pentosidine, are formed by an oxidative process, and often referred to as glycoxidation products. AGEs have been reported to accumulate during chronological aging but sun exposure has also been shown to contribute to this accumulation likely through induction of an oxidative environment. The purpose of this study was to investigate the accumulation of AGEs in human skin and, more specifically, CML and pentosidine, both of which are generated by oxidative pathways.
\end{abstract}

Methods: CML and pentosidine immunolabelling were investigated in skin samples from both non photoexposed (sun protected) and photo-exposed (sun exposed) sites from donors of two different age groups (18-25 years and $70-75$ years).

Results: Results demonstrate CML and pentosidine accumulation in sun-exposed skin especially in the aged group. A vicious circle is envisioned in which the presence of AGEs in a tissue accelerates the formation of additional glycoxidation products following UV-exposure.

Conclusion: The damaging effects of UV radiation might be more detrimental in aged skin than in young skin due, in part, to an increased accumulation of AGEs and, in turn, the exacerbation of alterations caused by chronological aging.

Keywords: CML; Pentosidine; Glycation; Glycoxidation; Aging skin; Ultraviolet exposure

\section{Introduction}

Skin aging is the result of intrinsic and extrinsic phenomena inducing important alterations in morphological, physical and biological properties of the tissue [1]. Among the factors involved in skin aging, the glycation reaction is an important element. Briefly, this non enzymatic reaction, also known as the Maillard reaction, takes place between free amino groups in proteins (lysine and arginine side groups) and a reducing sugar such as glucose and leads to the formation of advanced glycation end-products (AGEs) [2]. Oxidative environments resulting in the formation of oxoaldehydes, metalcatalyzed auto-oxidation of reducing sugars, and auto-oxidation of Amadori Products induce AGEs formation [3]. AGEs produced by oxidative pathways are referred to as glycoxidation products [4]. Many AGEs have been described and identified in various human tissues including human skin e.g. N-e-carboxymethyl-lysine (CML) [5], pentosidine [6-9] (Figure 1). Of the many skin proteins found in the skin, dermal proteins making up the extracellular matrix (ECM) with a long half life and a slow turnover, such as collagen, may be particularly affected in vivo by this process [10].

Accumulation of AGEs with age is well-known, notably in skin and is believed to modify skin homeostasis [11]. For example, AGEs have been shown to alter fibroblast viability by inducing the apoptotic process $[12,13]$, a senescence phenotype [14] or a cytotoxic effect [15]. Glycation products have also been reported to alter the biology of these dermal cells by disturbing the balance between synthesis of extracellular matrix components and enzymes [16-18] and consequently skin structure. Most of these biological responses could be mediated by Receptor of AGE (RAGE); the AGE-RAGE interaction alters cellular signaling, alters gene expression, enhances the release of pro-inflammatory cytokines and increases intracellular reactive oxygen species (ROS) via

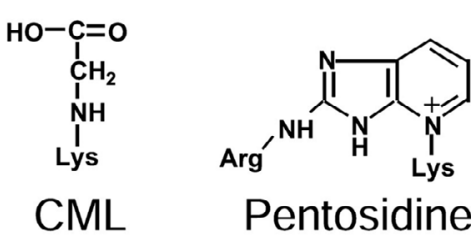

Figure 1: Structures of CarboxyMethyl-Lysine (CML) and pentosidine.

NADPH-oxidase [19]. If biological signature is altered, the mechanical properties are also disrupted [20] and could explain the loss of elasticity observed with age [21].

Skin photo aging is the result of a cumulative process wherein UVinduced damage is superimposed on intrinsic (chronological) skin aging [1]. Particularly, it has been reported that dermal extracellular matrix can be altered by UVA. It has been suggested that cross-linked structures in dermal ECM could act as photo-sensitizers for UVA-induced oxidative damage in skin [22]. Wondrak et al. described endogenous chromophores in human skin as photosensitizers: following absorption

*Corresponding author: Pageon H, L'Oréal Research \& Innovation, 1 Avenue Eugène Schueller 93600 Aulnay-Sous-Bois, France, Tel: 33-1-48-68-92-42; E-mail : hpageon@rd.loreal.com

Received November 08, 2013; Accepted November 18, 2013; Published November 25, 2013

Citation: Pageon H, Poumès-Ballihaut C, Zucchi H, Bastien P, Tancrede E Asselineau D (2013) Aged Human Skin is More Susceptible than Young Skin to Accumulate Advanced Glycoxidation Products Induced by Sun Exposure. Aging Sci 1: 112. doi: 10.4172/2329-8847.1000112

Copyright: $\odot 2013$ Pageon $\mathrm{H}$, et al. This is an open-access article distributed unde the terms of the Creative Commons Attribution License, which permits unrestricted use, distribution, and reproduction in any medium, provided the original author and source are credited. 
Citation: Pageon H, Poumès-Ballihaut C, Zucchi H, Bastien P, Tancrede E, Asselineau D (2013) Aged Human Skin is More Susceptible than Young Skin to Accumulate Advanced Glycoxidation Products Induced by Sun Exposure. Aging Sci 1: 112. doi: 10.4172/2329-8847.1000112

Page 2 of 5

of solar photons (particularly in the UVA region), these photoexcited components generate ROS, organic free radicals and other toxic photoproducts that mediate skin photo-oxidative stress. Among these components, AGEs have been identified as putative photosensitizers [23]. Following UVA-exposure of pentosidine-rich compounds, hydrogen peroxide $\left(\mathrm{H}_{2} \mathrm{O}_{2}\right)$ production is increased at a pentosidine concentration-dependent level [24]. Chronic sunlight exposure of the skin induces hyperplasia of elastic tissue in the upper dermis known as actinic elastosis. It has been reported that lesions of actinic elastosis accumulate CML and that ultraviolet-induced oxidation may accelerate CML formation in this case [25]. Jeanmaire et al. illustrated this result by comparing CML accumulation in photo-exposed (glabella) and non photo-exposed (breast) skin from the same donor. They observed higher CML accumulation in the exposed site. Using a 3D-skin model of de-epidermized dermis (DED), they demonstrated in vitro that UVexposure of the DED skin model potentiated glycation [26].

The purpose of our study was to investigate AGEs accumulation, and more specifically CML and pentosidine in human skin. We compared skin specimens from both non photo-exposed and photoexposed sites from young and old donors.

\section{Materials and Methods}

\section{Skin samples}

Skin samples from two age groups (young $=18-25$ years, $N=6$ and old $=70-75$ years, $N=7$ ) of healthy volunteers were analyzed. They were all non-smoking females and Fitzpatrick phototypes between II and IV.

Punch biopsies ( $3 \mathrm{~mm}$ in diameter) were collected from inner (non photo-exposed) and dorsal (photo-exposed) forearms of each subject after approval by an ethics committee and informed written consent.

\section{Histological staining}

Skin biopsies were fixed in neutral formalin and processed for histology. Paraffin sections $(5 \mu \mathrm{m})$ were processed and stained with Hematoxylin Eosin Safron (HES) and Orcein for elastic fibers.

\section{Immunolabelling}

Immunolabelling was performed on the $5 \mu \mathrm{m}$ paraffin sections using mouse monoclonal antibodies against CML (clone CMS-10 from Transgenic reference KH011, dilution 1:50) and pentosidine (clone PEN-12 from Transgenic reference KH012, dilution 1:150). Immunolabelling was performed overnight at room temperature with an amplificatory system Vectastain RTU Universal VECTOR and developed in VIP (kit Vector SK4600).

Immunolabelled tissue sections were observed and imaged under transmission optic microscope (DMR, Leica, Microsystems). Quantitative image analysis was performed using Histolab software (version7.6.0) from Microvision Instruments (Evry, France). Quantification was performed only on the dermal extracellular matrix and cells were excluded. The ratio between the total surface of dermis and the positive surface occupied by the AGEs after immunostaining is reported.

\section{Statistical methodology}

CML and pentosidine expression levels are described by skin type (old versus young) and site (exposed versus non-exposed) using box plots. The bottom and the top of the box represent the first and third quartiles. The horizontal band and the diamond inside the box represent the median and the mean value, respectively. Individual values have been superposed for completeness. Young vs old donors were compared by zone using exact Wilcoxon tests. Exposed vs unexposed sites were compared according to age group (old vs young) using paired Wilcoxon signed rank tests. All statistical analyses were performed using SAS 9.3 statistical software. The two-sided significance threshold was set at $5 \%$.

\section{Results}

\section{Skin morphology}

Hematoxylin eosin safron (HES) and orcein staining confirmed the presence of known age-induced skin alterations: decreased epidermal and dermal thickness, flattening of the dermal epidermal junction (DEJ) and elastosis, in particular on the sun-exposed site (Figure 2).

\section{CML and pentosidine in skin samples}

Difference between non photo-exposed and photo-exposed sites in "young" skin samples: CML or pentosidine formation in forearm skin of the young group did not vary according to sun exposure (Figure $3 \mathrm{~A}, 3 \mathrm{~B}, 3 \mathrm{E}$ and $3 \mathrm{~F}$ ). Indeed, no modifications were observed when comparing inner (Figure $3 \mathrm{~A}$ and $3 \mathrm{E}$ ) and dorsal (Figure $3 \mathrm{~B}$ and $3 \mathrm{~F}$ ) sites of forearm skin. Image analysis showed no statistically significant differences between both areas (Figure 4A and 5A Inner and Dorsal “young" box plot).

Difference between non photo-exposed and photo-exposed in "old" skin specimens: In contrast, CML and pentosidine formation in skin of the older group augmented in sun-exposed skin (Figure 3C, $3 \mathrm{D}, 3 \mathrm{G}$ and $3 \mathrm{H}$ ). Increased positive immunostaining was observed in dorsal (Figure 3D and $3 \mathrm{H}$ ) compared to inner (Figure 3C and 3G) forearm sites. Image analysis showed a statistically significant difference for pentosidine (Figure 5A Inner and Dorsal "old" box plot, $\mathrm{p}=0.0156$ ) and a tendency $(\mathrm{p}=0.0781)$ towards increased CML immunostaining (Figure 4A Inner and Dorsal "old” boxplots).

Comparison of "young" and "old" skin specimens: An accumulation of AGEs was observed in older skin as compared to young skin (Figure 3). A statistical difference was observed for both pentosidine $(\mathrm{p}=0.0012$; Figure $3 \mathrm{~F}$ and $3 \mathrm{H})$ and $\mathrm{CML}(\mathrm{p}=0.0066$; Figure $3 \mathrm{~B}$ and $3 \mathrm{D})$ in dorsal skin as well as for pentosidine $(\mathrm{p}=0.0012$; Figure $3 \mathrm{E}$ and $3 \mathrm{G}$ ) in inner skin. A tendency towards a CML (Figure $3 \mathrm{~A}$ and $3 \mathrm{C}$ ) increase was observed in inner forearm skin. As noted above, sun exposure in older human skin, increased the accumulation of CML $(\mathrm{x} 1.5, \mathrm{~ns} \mathrm{p}=0.0781)$ and pentosidine $(\mathrm{x} 2, \mathrm{p}=0.0156)$ as showed in the
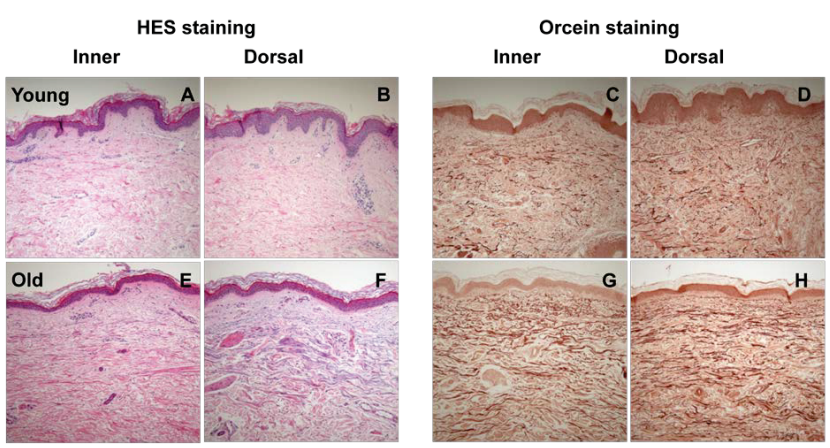

Figure 2: Histologic staining of human skin specimens.

Representative skin morphology using Hematoxylin Eosin Safron (HES) staining $(A, B, E, F)$ and elastic fibers using orcein staining $(C, D, G, F)$ of human skin specimens from inner forearm (non photo-exposed) (A, E, C, G) or dorsal forearm (photo-exposed) $(B, F, D, H)$ of "young" (A-D) or "old" $(E-H)$ donors. 
Citation: Pageon H, Poumès-Ballihaut C, Zucchi H, Bastien P, Tancrede E, Asselineau D (2013) Aged Human Skin is More Susceptible than Young Skin to Accumulate Advanced Glycoxidation Products Induced by Sun Exposure. Aging Sci 1: 112. doi: 10.4172/2329-8847.1000112

Page 3 of 5

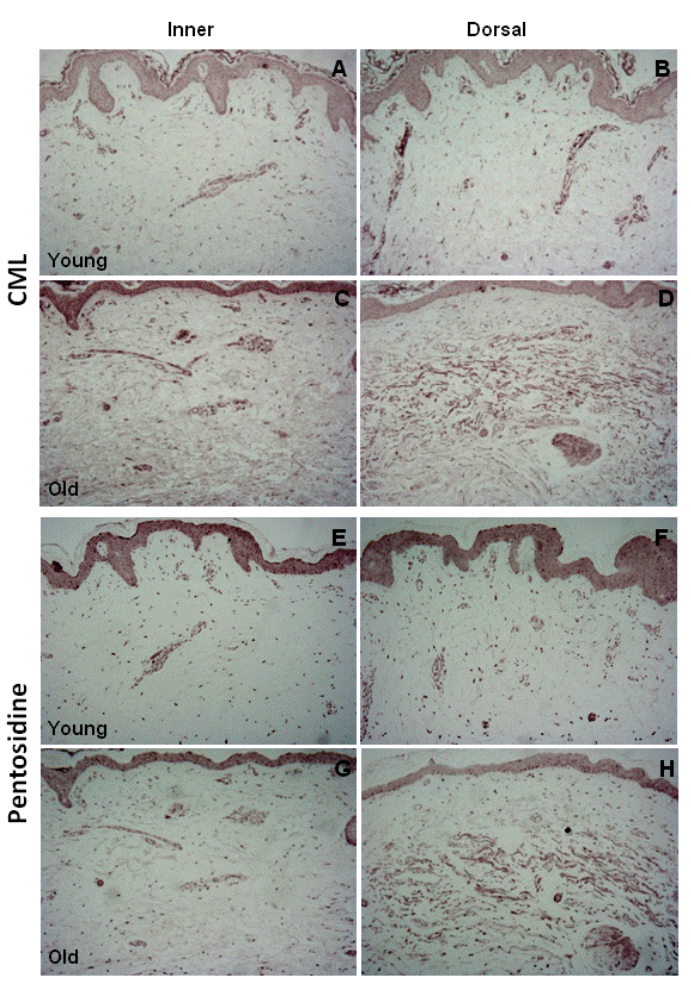

Figure 3: CML and Pentosidine staining

Representative Immunolabelling of CML (A-D) and pentosidine $(\mathrm{E}-\mathrm{H})$ of human skin specimens from inner (non photo- exposed) $(A, C, E, G)$ or dorsal forearm (photo-exposed) (B, D, F, H) of "young" (A, B, E, F) or "old" (C, D, G, H) donors. Note that CML and pentosidine were strongly detected in "old" human skin specimens as compared to "young" skin specimens and were potentiated with solar exposure in the former group.

Dorsal/Inner ratio in Figure $4 \mathrm{~B}$ and $5 \mathrm{~B}$ whereas no such effect was detected in "young" skin specimens.

\section{Discussion}

Human skin aging results in decreased elasticity, accumulation of insoluble collagen and impaired wound healing, phenomena which may be explained, in part, by AGEs formation. The protein turn-over rate is important in AGEs accumulation in tissues. In skin, the halflife of collagen has been reported to be fifteen years and consequently this molecule is highly susceptible to AGEs accumulation [5]. Verzijl et al. [10] showed, in normal buttock skin (photo protected) of subjects from 19 to 91 years of age, that CML and pentosidine accumulated linearly with chronological age. Additional reports have also described an increase in these AGEs with solar exposure $[25,26]$. We show here that sun exposure of the skin increases the abundance of AGEs, specifically CML and pentosidine, and not only in the areas affected by actinic elastosis. We observed age-related AGEs accumulation in the inner face of the forearm and an even higher level in the photo-exposed site (dorsal side of the forearm) of older donors. We believe that AGEs produced by chronological aging may act as photosensitizers [23] and act as an important source of ROS in the dermal matrix facilitating the formation of additional glycoxidation products, such as CML and pentosidine following UV exposure. In support of this theory, Okano et al. demonstrated that pentosidine itself is photo-unstable and produces hydrogen peroxide in the medium after UVA exposure [24]. In addition, oxidative environments have been shown to create a toxic effect on cell viability in the presence of AGEs as shown by Mazaki on human dermal fibroblasts [27].

The consequences of AGEs accumulation in the skin are likely to be many. Pentosidine cross-linking of proteins chains may be partly responsible for the impairment of skin biomechanical properties [20] and elasticity [21] that has reported with age. CML-collagen has also been reported to induce a time-dependent and dose-dependent increase in fibroblast apoptosis and this process has been shown to be mediated by RAGE [12]. RAGE is highly expressed in skin and upregulated in sun-exposed skin [28]. The expression of RAGE and AGEs interaction has been reported to increase gene transcription of proinflammatory and pro-fibrotic cytokines, chemokines [19] and to be implicated in melanoma process and metastasis [29]. In consequence, AGEs accumulation in the skin increases the possible interaction with RAGE and the following deleterious effects on cells and extracellular matrix. Many studies reported AGEs effects on extracellular matrix maturation and quality through alterations of macromolecules and synthesis of degradation enzymes [11].
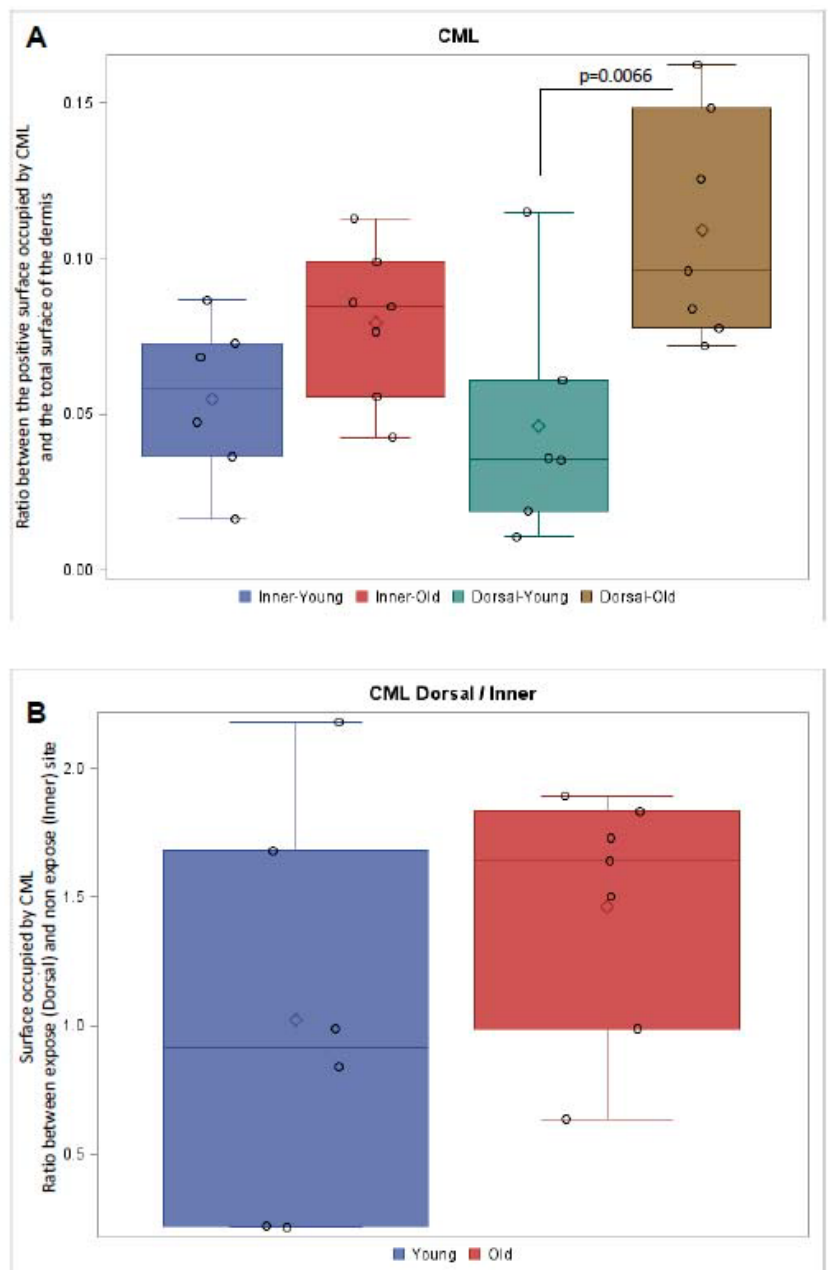

Figure 4: $\mathrm{CML}$ values, represented as histograms, correspond to the surface area occupied by CML for each considered group (A) Inner-Young (blue) Dorsal-Young (Red) Inner-Old (Green) Dorsal-Old (Brown) and ratio between Dorsal and Inner sites (B) for "Young" (blue) and "Old" (red) groups. Note that $C M L$ was detected in old human skin specimens as compared to young skin specimen (but seems to be only a trend $p=0.1375$ ) and was potentiated by solar exposure in this group $(p=0.0781)$. 
Citation: Pageon H, Poumès-Ballihaut C, Zucchi H, Bastien P, Tancrede E, Asselineau D (2013) Aged Human Skin is More Susceptible than Young Skin to Accumulate Advanced Glycoxidation Products Induced by Sun Exposure. Aging Sci 1: 112. doi: 10.4172/2329-8847.1000112

Page 4 of 5
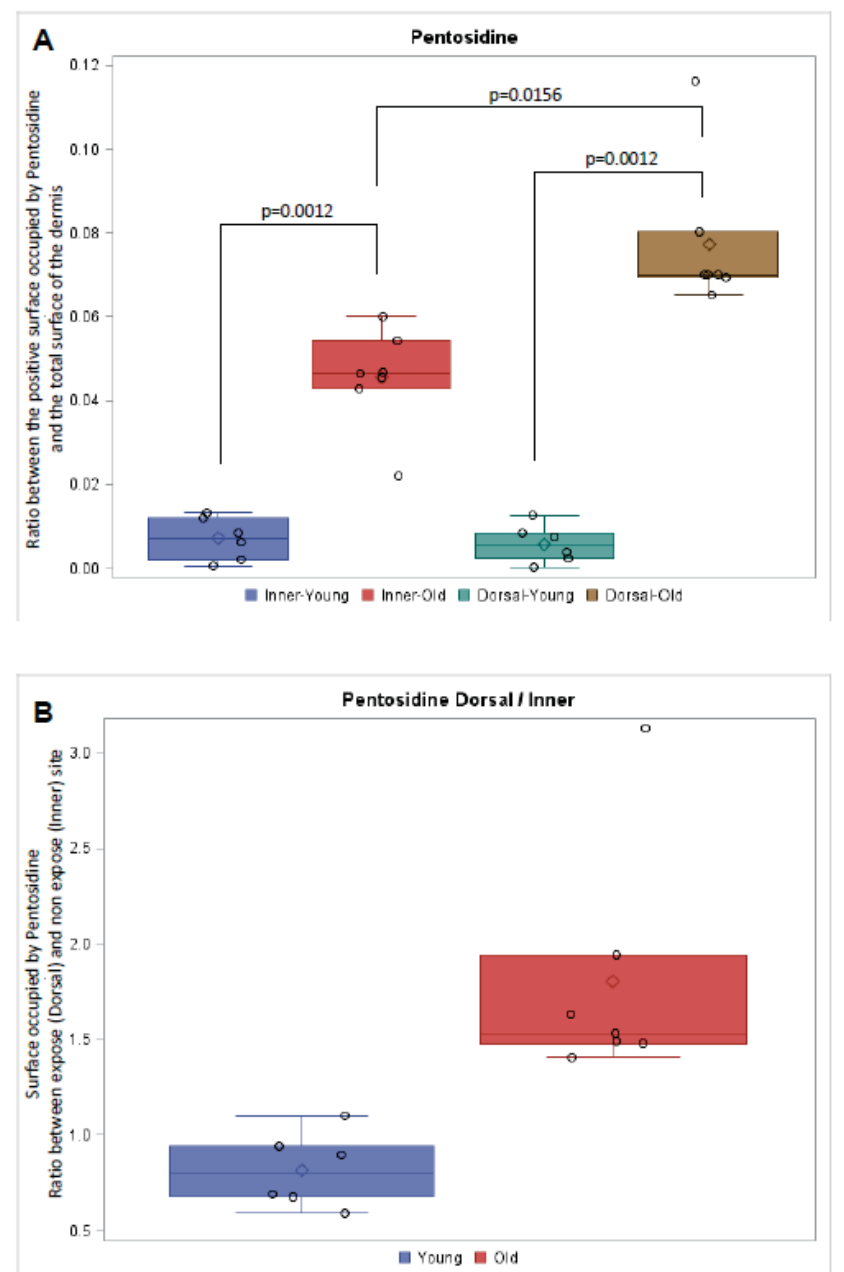

Figure 5: Pentosidine values, represented as histograms, correspond to the surface area occupied by pentosidine for each considered group (A) InnerYoung (blue) Dorsal-Young (Red) Inner-Old (Green) Dorsal-Old (Brown) and ratio between Dorsal and Inner sites (B) for Young (blue) and Old (red) groups. Note that pentosidine was strongly detected in "old" human skin specimens as compared to "young" skin specimens $(p=0.0012)$ and was potentiated by solar exposure in this group $(p=0.0156)$.

Our results demonstrate the role of solar exposure in CML and pentosidine accumulation with aging. This accumulation may be due to repeated exposure over time but we also believe that the presence of AGEs in older skin specimens may actively promote increased accumulation of glycoxidation products such as CML and pentosidine. A vicious circle is envisioned in which the presence of AGEs in a tissue accelerates the formation of additional AGEs following UV-exposure. We thus speculate that the damaging effects of sun exposure are more adverse in aged skin than in young skin through an enhancement of toxic effect on cells and extracellular matrix and an exacerbation of agerelated alterations.

Consequently, the use of molecules with antioxidant activity may help to inhibit or slow down the accumulation of glycoxydation products induced by photo exposure. Previously, we demonstrated the antiglycation effect of a blueberry extract [30] which, indeed, has powerful antioxidant properties due to a rich polyphenols, flavonoïds and anthocyanins [31] content. Such ingredients are promising ingredients for minimizing formation of glycoxidation products and for combating skin aging processes.

\section{Acknowledgements}

We would like to thank Dr Michelle Rathman-Josserand for critical reading of this manuscript.

\section{References}

1. Zouboulis CC, Makrantonaki E (2011) Clinical aspects and molecular diagnostics of skin aging. Clin Dermatol 29: 3-14.

2. Ulrich P, Cerami A (2001) Protein glycation, diabetes, and aging. Recent Prog Horm Res 56: 1-21.

3. Tessier FJ (2010) The Maillard reaction in the human body. The main discoveries and factors that affect glycation. Pathol Biol (Paris) 58: 214-219.

4. Dyer DG, Blackledge JA, Katz BM, Hull CJ, Adkisson HD, et al. (1991) The Maillard reaction in vivo. Z Ernahrungswiss 30: 29-45.

5. Dunn JA, Patrick JS, Thorpe SR, Baynes JW (1989) Oxidation of glycated proteins: age-dependent accumulation of $\mathrm{N}$ epsilon-(carboxymethyl) lysine in lens proteins. Biochemistry 28: 9464-9468.

6. Sell DR, Monnier VM (1989) Structure elucidation of a senescence cross-link from human extracellular matrix. Implication of pentoses in the aging process. J Biol Chem 264: 21597-21602.

7. Sell DR, Nagaraj RH, Grandhee SK, Odetti P, Lapolla A, et al. (1991) Pentosidine: a molecular marker for the cumulative damage to proteins in diabetes, aging, and uremia. Diabetes Metab Rev 7: 239-251.

8. Dyer DG, Blackledge JA, Thorpe SR, Baynes JW (1991) Formation of pentosidine during nonenzymatic browning of proteins by glucose. Identification of glucose and other carbohydrates as possible precursors of pentosidine in vivo. J Biol Chem 266: 11654-11660.

9. Dyer DG, Dunn JA, Thorpe SR, Bailie KE, Lyons TJ, et al. (1993) Accumulation of Maillard reaction products in skin collagen in diabetes and aging. $J$ Clin Invest 91: 2463-2469.

10. Verzijl N, DeGroot J, Thorpe SR, Bank RA, Shaw JN, et al. (2000) Effect of collagen turnover on the accumulation of advanced glycation end products. $J$ Biol Chem 275: 39027-39031.

11. Pageon $H$ (2010) Reaction of glycation and human skin: the effects on the skin and its components, reconstructed skin as a model. Pathol Biol (Paris) 58: 226-231.

12. Alikhani Z, Alikhani M, Boyd CM, Nagao K, Trackman PC, et al. (2005) Advanced glycation end products enhance expression of pro-apoptotic genes and stimulate fibroblast apoptosis through cytoplasmic and mitochondrial pathways. J Biol Chem 280:12087-12095.

13. Alikhani M, Maclellan CM, Raptis M, Vora S, Trackman PC, et al. (2007) Advanced glycation end products induce apoptosis in fibroblasts through activation of ROS, MAP kinases, and the FOXO1 transcription factor. Am J Physiol Cell Physiol 292: C850- C856.

14. Ravelojaona V, Robert AM, Robert L (2009) Expression of senescenceassociated beta galactosidase (SA-beta-Gal) by human skin fibroblasts, effect of advanced glycation end-products and fucose or rhamnose-rich polysaccharides. Arch Gerontol Geriatr 48: 151-154.

15. Ravelojaona V, Péterszegi G, Molinari J, Gesztesi JL, Robert L (2007) Demonstration of the cytotoxic effect of Advanced Glycation Endproducts (AGE-s). J Soc Biol 201: 185-188.

16. Okano Y, Masaki H, Sakurai H (2002) Dysfunction of dermal fibroblasts induced by advanced glycation end-products (AGEs) and the contribution of a nonspecific interaction with cell membrane and AGEs. J Dermatol Sci 29: 171-180.

17. Molinari J, Ruszova E, Velebny V, Robert L (2008) Effect of advanced glycation end products on gene expression profiles of human dermal fibroblasts. Biogerontology 9: 177-182.

18. Pageon H, Bakala H, Monnier VM, Asselineau D (2007) Collagen glycation triggers the formation of aged skin in vitro. Eur J Dermatol 17: 12-20.

19. Barlovic DP, Soro-Paavonen A, Jandeleit-Dahm KA (2011) RAGE biology, atherosclerosis and diabetes. Clin Sci (Lond) 121: 43-55. 
Citation: Pageon H, Poumès-Ballihaut C, Zucchi H, Bastien P, Tancrede E, Asselineau D (2013) Aged Human Skin is More Susceptible than Young Skin to Accumulate Advanced Glycoxidation Products Induced by Sun Exposure. Aging Sci 1: 112. doi: 10.4172/2329-8847.1000112

Page 5 of 5

20. Reihsner R, Melling M, Pfeiler W, Menzel EJ (2000) Alterations of biochemical and two-dimensional biomechanical properties of human skin in diabetes mellitus as compared to effects of in vitro non-enzymatic glycation. Clin Biomech (Bristol, Avon) 15: 379-386.

21. Corstjens H, Dicanio D, Muizzuddin N, Neven A, Sparacio R, et al. (2008) Glycation associated skin autofluorescence and skin elasticity are related to chronological age and body mass index of healthy subjects. Exp Gerontol 43: 663-667.

22. Ou-Yang H, Stamatas G, Kollias N (2009) Dermal contributions to UVA-induced oxidative stress in skin. Photodermatol Photoimmunol Photomed 25: 65-70.

23. Wondrak GT, Jacobson MK, Jacobson EL (2006) Endogenous UVAphotosensitizers: mediators of skin photodamage and novel targets for skin photoprotection. Photochem Photobiol Sci 5: 215-237.

24. Okano Y, Masaki H, Sakurai H (2001) Pentosidine in advanced glycation endproducts (AGEs) during UVA irradiation generates active oxygen species and impairs human dermal fibroblasts. J Dermatol Sci 27: S11-S18.

25. Mizutari K, Ono T, Ikeda K, Kayashima K, Horiuchi S (1997) Photo-enhanced modification of human skin elastin in actinic elastosis by $\mathrm{N}$ (epsilon)(carboxymethyl)lysine, one of the glycoxidation products of the Maillard reaction. J Invest Dermatol 108: 797-802.
26. Jeanmaire C, Danoux L, Pauly G (2001) Glycation during human derma intrinsic and actinic ageing: an in vivo and in vitro model study. $\mathrm{Br} \mathrm{J}$ Dermatol 145: 10-18.

27. Masaki $H$, Okano $Y$, Sakurai $H$ (1999) Generation of active oxygen species from advanced glycation end-products (AGEs) during ultraviolet light A (UVA) irradiation and a possible mechanism for cell damaging. Biochim Biophys Acta 1428: 45-56.

28. Lohwasser C, Neureiter D, Weigle B, Kirchner T, Schuppan D (2006) The receptor for advanced glycation end products is highly expressed in the skin and upregulated by advanced glycation end products and tumor necrosis factor-alpha. J Invest Dermatol 126: 291-299.

29. Abe $R$, Shimizu T, Sugawara $H$, Watanabe $H$, Nakamura $H$, et al. (2004) Regulation of human melanoma growth and metastasis by AGE-AGE receptor interactions. J Invest Dermatol 122: 461-467.

30. Pageon H, Técher MP, Asselineau D (2008) Reconstructed skin modified by glycation of the dermal equivalent as a model for skin aging and its potential use to evaluate anti-glycation molecules. Exp Gerontol 43: 584-588.

31. Faria A, Oliveira J, Neves P, Gameiro P, Santos-Buelga C, et al. (2005) Antioxidant properties of prepared blueberry (Vaccinium myrtillus) extracts. $J$ Agric Food Chem 53: 6896-6902. 